\title{
Electron Microscopy with Structured Electrons
}

\author{
Benjamin J. McMorran ${ }^{1}$, Peter Ercius ${ }^{2}$, Tyler R. Harvey ${ }^{1}$, Martin Linck ${ }^{3}$, Colin Ophus ${ }^{4}$, Jordan Pierce ${ }^{1}$ \\ 1. Department of Physics, University of Oregon, Eugene, Oregon, USA. \\ 2. National Center for Electron Microscopy, Molecular Foundry, Lawrence Berkeley National \\ Laboratory, Berkeley, California, USA. \\ 3. Corrected Electron Optical Systems GmbH, Heidelberg, Germany.
}

Nanofabricated diffractive optics are new tools for coherently dividing and manipulating electron wavefunctions, similar to how spatial light modulators are used to engineer light waves in optics. For example, diffraction holograms can be used in a scanning transmission electron microscope to remove aberrations [1], implement new types of interferometric phase contrast [2,3], and produce electron probes with phase vortices and quantized angular momentum [4,5].

We are currently investigating the use of nanoscale gratings for off-axis STEM interferometry (Figure 1). We have used a single grating in a scanning transmission electron microscope (STEM) to form a pathseparated electron interferometer [6]. In this configuration, single electrons are coherently divided before the specimen, and then focused into two or more focused probes at the specimen. We are developing ways to use this for electron interferometry and STEM-holography [7]. Advantages of using a grating as an amplitude-type beamsplitters over Moellenstedt biprisms in conventional electron holography is that (a) it preserves the symmetry of the probe, essentially creating multiple copies of it, (b) it loosens the coherent requirements of the initial electron beam, potentially enabling electron holography in an expanded range of instruments, (c) eliminates the voltage stability requirements of a biprism, and (d) allows for structured probes by incorporating additional holographic features into the grating.

Nanostructured electron optics also can be used to implement new types of on-axis interferometric phase contrast in STEM (Figure 2). For example, in Matched Illumination-Detector Interferometry STEM (MIDI-STEM), a transverse phase modulation is introduced to a STEM probe using a nanostructured phase plate in the probe-defining condenser aperture [2,3]. The diffraction plane of the probe is imaged at each probe position. Virtual detector zones are defined on the fast imaging detector. Nonuniform phase introduced by the specimen across the probe results in intensity shifts between the pre-defined zones. For an appropriately defined phase plate, this can provide linear phase contrast at high resolutions in aberration-corrected STEM.

\section{References:}

1. B. McMorran, M. Linck, P. Moeck, 9,240,255 (11 September 2014).

2. C. Ophus, J. Ciston, J. Pierce, T. R. Harvey, J. Chess, B. J. McMorran, C. Czarnik, H. H. Rose, P. Ercius, Nat. Commun. 7, 10719 (2016).

3. C. Ophus, J. Ciston, H. Yang, J. Pierce, T. T. Harvey, J. Chess, B. J. McMorran, C. Czarnik, H. Rose, and P. Ercius, Microsc. Microanal. 22, 460 (2016). 
4. B. J. McMorran, A. Agrawal, I. M. Anderson, A. A. Herzing, H. J. Lezec, J. J. McClelland, J. Unguris, Science 331, 192 (2011).

5. B. J. McMorran, A. Agrawal, P. A. Ercius, V. Grillo, A. A. Herzing, T. R. Harvey, M. Linck, J. S. Pierce, "Origins and demonstrations of electrons with orbital angular momentum," Phil. Trans. R. Soc. A 375, 20150434 (2017).

6. F. S. Yasin, T. R. Harvey, J. J. Chess, J. S. Pierce, and B. J. McMorran, "Development of STEMHolography," Microsc. Microanal. 22, 506 (2016).

7. J. M. Cowley, Ultramicroscopy 96, 163-166 (2003).

8. Work at the Molecular Foundry was supported by the Office of Science, Office of Basic Energy Sciences, of the U.S. Department of Energy under Contract No. DE-AC02-05CH11231. Work at University of Oregon was supported by the U.S. DOE under Award No. DE-SC0010466.
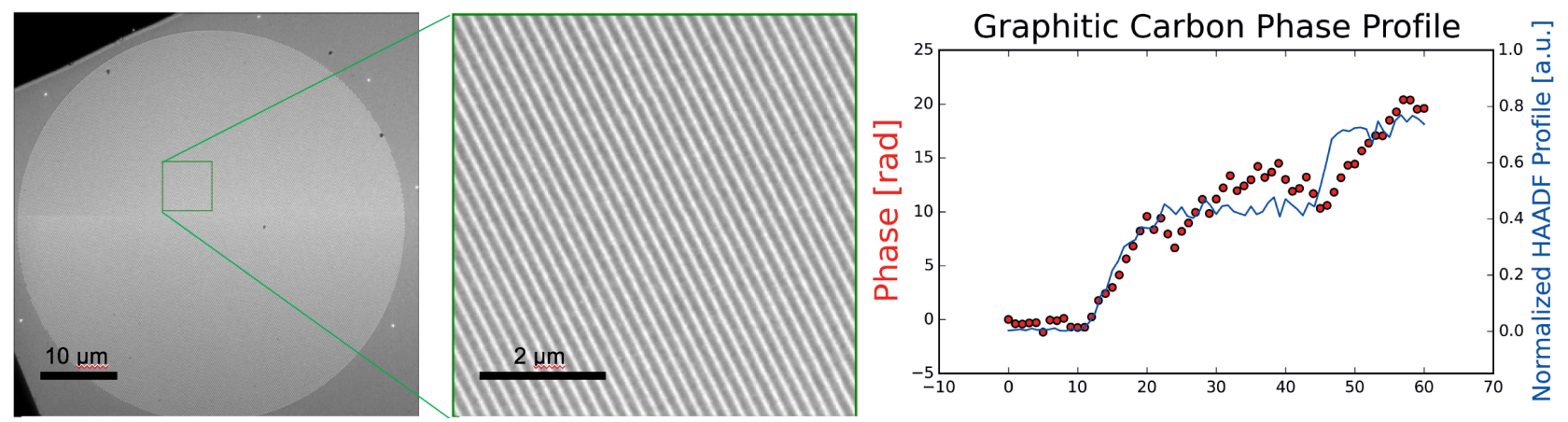

Figure 1. A single grating (left image) placed in the condenser aperture of a STEM creates multiple probes at the specimen plane separated by $50 \mathrm{~nm}$ or more. Post-specimen imaging optics form an interference pattern between the multiple probes (center). When one of the probes passes through a specimen and acquires a phase, this interference pattern shifts and evolves. An analysis of the interference at each probe position can be used to extract the phase introduced by a transparent specimen (right).
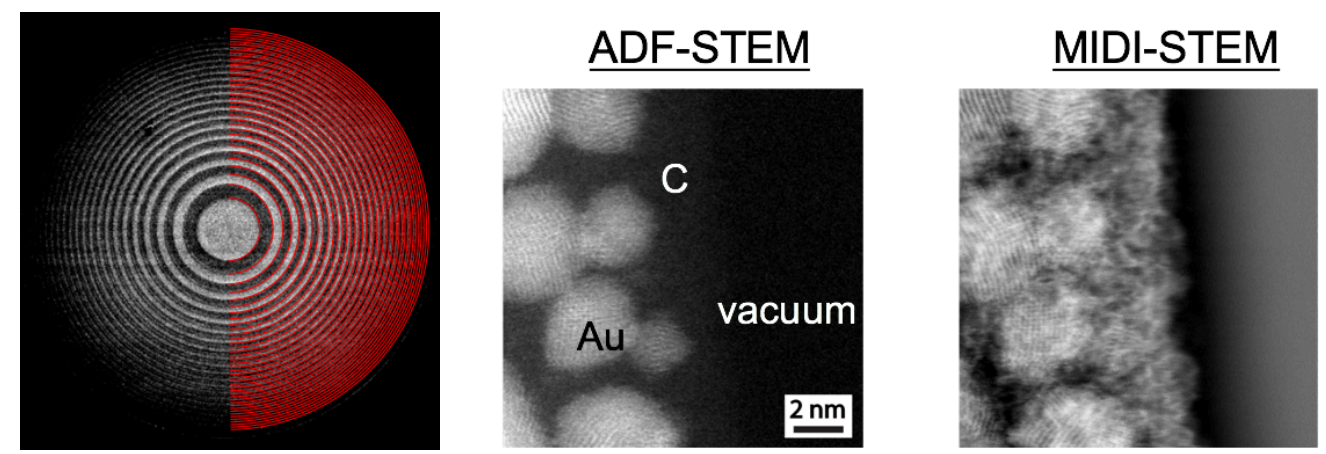

Figure 2. In MIDI-STEM, a structured electron beam is used to provide linear phase contrast. (Left) A phase plate in the condenser aperture introduces a binary phase structure to the electron beam, creating alternating areas of light and dark areas in the back focal plane of the focused STEM probe. A fast imaging detector (Gatan K2) images this at every probe position. Shifts in the difference between total counts in the two sets of areas (delineated here by red lines) is used to form the signal. Conventional ADF-STEM image shows little phase contrast (center) compared to the MIDI-STEM technique (right). 